\title{
Neonatal Spell
}

National Cancer Institute

\section{Source}

National Cancer Institute. Neonatal Spell. NCI Thesaurus. Code C118509.

Cardiorespiratory events that are characterized by variable combinations of cessation of breathing, decrease in blood oxygen saturation, and decreased heart rate. 